\title{
HSV-1 interaction to 3-O-sulfated heparan sulfate in mouse-derived DRG explant and profiles of inflammatory markers during virus infection
}

\author{
Harsh Sharthiya ${ }^{1}$ - Chanmoly Seng ${ }^{2}$ - T. H Van Kuppevelt ${ }^{3}$ - Vaibhav Tiwari ${ }^{4}$. \\ Michele Fornaro ${ }^{1}$ (D)
}

Received: 8 December 2016 / Revised: 25 January 2017 / Accepted: 9 February 2017 / Published online: 21 March 2017

(C) The Author(s) 2017. This article is published with open access at Springerlink.com

\begin{abstract}
The molecular mechanism of herpes simplex virus (HSV) entry and the associated inflammatory response in the nervous system remain poorly understood. Using mousederived ex vivo dorsal root ganglia (DRG) explant model and single cell neurons ( $\mathrm{SCNs}$ ), in this study, we provided a visual evidence for the expression of heparan sulfate (HS) and 3-O-sulfated heparan sulfate (3-OS HS) followed by their interactions with HSV-1 glycoprotein $\mathrm{B}(\mathrm{gB})$ and glycoprotein $\mathrm{D}(\mathrm{gD})$ during cell entry. Upon heparanase treatment of DRGderived SCN, a significant inhibition of HSV-1 entry was observed suggesting the involvement of HS role during viral entry. Finally, a cytokine array profile generated during HSV-1 infection in DRG explant indicated an enhanced expression of chemokines (LIX, TIMP-2, and M-CSF) — known regulators of HS. Taken together, these results highlight the significance of HS during HSV-1 entry in DRG explant. Further investigation is needed to understand which isoforms of 3-O-
\end{abstract}

Electronic supplementary material The online version of this article (doi:10.1007/s13365-017-0521-4) contains supplementary material, which is available to authorized users.

Vaibhav Tiwari

vtiwar@midwestern.edu

Michele Fornaro

mforna@midwestern.edu

1 Department of Anatomy, Chicago College of Osteopathic Medicine, Midwestern University, Downers Grove, IL 60515, USA

2 Department of Biomedical sciences, College of Health Sciences, Midwestern University, Downers Grove, IL 60515, USA

3 Department of Biochemistry, Nijmegen Institute for Molecular Life Sciences, Radboud University, 6500 HB Nijmegen, The Netherlands

4 Department of Microbiology and Immunology, Chicago College of Osteopathic Medicine, Midwestern University, Downers Grove, IL 60515, USA sulfotransferase (3-OST)-generated HS contributed during HSV-1 infection and associated cell damage.

Keywords HSV entry · Heparan sulfate · Sensory neurons · Neuroinflammation

\section{Introduction}

The hallmark of herpes simplex virus type 1 (HSV-1) infection is to establish life-long latency in the sensory ganglia mainly in the dorsal root ganglia (DRG) of the host following an initial infection in epithelial cells (Roizman and Whitley 2013; Fraser and Valyi-Nagy 1993; Wilson and Mohr 2012). Upon reactivation, the virus resumes its cycle of lytic gene expression to replicate successfully and transport newly made virions axonally back either to peripheral nervous system, resulting in cold sores or fever blisters and corneal keratitis, or central nervous system resulting in meningitis and encephalitis in the brain (Whitley and Roizman 2001; Nicoll et al. 2012; Simmons 2002).

The process of HSV-1 entry initiates with the specific interaction between viral envelope glycoproteins and host cell surface receptors (Antoine et al. 2013; Spear 2004; Hadigal and Shukla 2013; Connolly et al. 2011). The HSV glycoproteins $\mathrm{B}$ and $\mathrm{C}$ ( $\mathrm{gB}$ and $\mathrm{gC}$, respectively) mediate their initial attachment to host cell surface heparan sulfate proteoglycans (HSPG) (Shukla and Spear 2001; Tiwari et al. 2015; WuDunn and Spear 1989; Herold et al. 1991; Shieh et al. 1992). The initial binding step of the virus to HSPG results in a conformational change that brings major viral glycoprotein $\mathrm{D}(\mathrm{gD})$ binding domain to interact with any given host cell receptors (nectin, HVEM, and 3-O-sulfated heparan sulfate; 3-OS HS). The later process involves three additional HSV glycoproteins, $\mathrm{gB}, \mathrm{gH}$, and $\mathrm{gL}$, and possibly an additional $\mathrm{gH}$ co-receptor, which trigger the fusion of the viral envelope with the 
plasma membrane of host cells (Geraghty et al. 1998; Montgomery et al. 1996; Shukla et al. 1999; Eisenberg et al. 2012).

Interestingly, recent studies widely documented the role of heparan sulfate (HS) and the HS-degrading enzyme heparanase in the pathological process including neuroinflammation (Zhang et al. 2014; Li and Vlodavsky 2009; Parish 2006; Hadigal et al. 2015). Therefore, understanding the involvement of HS and modified HS in HSV-1 entry, spread, and cell damage at the molecular level will aid in the development of novel strategies to prevent virus spread and associated inflammation (Joyce et al. 2005; Rusnati et al. 2005; Ferro et al. 2007; Tiwari et al. 2011; Raman et al. 2012). In this study, we investigated the interaction between HSV-1 glycoproteins and HS (Yabe and Maeda 2015) and modified 3-OS HS generated by the enzyme 3-O-sulfotransferase (3OST) (Yabe et al. 2005).

In order to achieve our goal, we developed a mousederived ex vivo DRG explant and single cell neuronal (SCN) cultures as a model to study HSV-1 entry. We observed the susceptibility and replication of KOS HSV-1 strain in the DRG explants. In addition, our data further provided the visual and quantitative evidence to support the significance of HS, 3-OS HS, in promoting HSV entry and inducing changes in the pattern of expression of unique sub-set of chemokines. Finally, we propose that DRG-based ex vivo model provides a unique platform to further investigate which form of HS is critical for viral entry and cell damage.

\section{Material and methods}

An overall experimental design is schematically described in the Fig. 1.

Harvesting of DRG In accordance with institutional review board-approved protocols (IACUC-Midwestern University), mouse-derived DRG explants were isolated from adult NIH/Swiss mice (Harlan Laboratories, Chicago, IL). Briefly, the animals were euthanized and the vertebral column was surgically dissected. The vertebral canal was exposed by performing a double cut on both sides of the vertebral bodies using fine scissors. The ventral access through the vertebral bodies did not interfere with the DRG located along the dorsal roots. DRGs were localized on both sides of the spinal cord, removed, and cleaned from excess of fibers and connective tissue. DRG explants were then plated ex vivo in 12-well plates pre-coated with matrigel (Sigma-Aldrich, St. Louis, MO) in serum-free medium (SFM) (Fueshko and Wray 1994) and maintained in culture conditions $\left(37^{\circ} \mathrm{C}\right.$ and $5 \%$ $\mathrm{CO}_{2}$ ) for up to 6 days. The medium was changed every $72 \mathrm{~h}$.
Generating single cell neurons from DRGs After collection, explants were immediately placed in F12 media (Gibco, Waltham, MA) containing collagenase $(1.25 \mathrm{mg} / \mathrm{ml})$ (SigmaAldrich, St. Louis, MO) and incubated $\left(37{ }^{\circ} \mathrm{C}\right.$ and $\left.5 \% \mathrm{CO}_{2}\right)$ for $45 \mathrm{~min}$. The collagenase step was repeated for another 45 min. Afterward, explants were incubated in F12 media containing trypsin $(0.025 \%$, Sigma-Aldrich, St. Louis, MO) mixture for $30 \mathrm{~min}$ followed by F12 media-containing FBS (Sigma-Aldrich, St. Louis, MO) for $15 \mathrm{~min}$. Thereafter, explants were washed three times with F12 media and mechanically dissociated with a glass pipette until the solution turned cloudy indicating a successful dissociation. Dissociated cells were filtered through a $0.22-\mu \mathrm{m}$ filter (BD Falcon, Franklin Lakes, NJ) and centrifuged (2400 rpm) for $2 \mathrm{~min}$. After removing the supernatant, the cells were resuspended in neurobasal media (Sigma-Aldrich, St. Louis, MO) containing B-27 supplement (Life Technologies, Carlsbad, CA), PSN antibiotics (Gibco, Waltham, MA), $0.5 \mathrm{mM}$ L-glutamine (Sigma-Aldrich, St. Louis, MO) and NGF $(5 \mathrm{ng} / \mathrm{ml}$, Alomone Labs, Jerusalem, Israel). The resuspended cells were plated on laminin-coated cover slides (Neuvitro, Vancouver, WA).

\section{HSV-1 infection in DRG explants}

Groups of three DRG explants/tubes were infected with HSV$1 \mathrm{gL} 86$ (10,000 virions) in serum-free media. After $12 \mathrm{~h}$ of infection at $37^{\circ} \mathrm{C}$, explants were washed with PBS, fixed, and permeabilized, using buffer followed by incubation with XGal (5-bromo-4-chloro-3-indolyl- $\beta$-D-galactosidase; Invitrogen) at $1.0 \mathrm{mg} / \mathrm{ml}$, which yields an insoluble bluestained product upon hydrolysis by $\beta$-galactosidase. Blue cells (representing viral entry) were seen at multiple locations per explant as shown in the supplementary figures (Fig. 1S). Microscopy was performed using Zeiss Axiovert 100.

\section{Plaque formation using infected DRGs}

A group of three DRG explants per well in triplicate experiment was cultured for 3 days prior to be infected with replication-competent syn mutant HSV-1(KOS-804) (Little and Schaffer 1981) or mock infected with PBS alone as a control for $2 \mathrm{~h}$ at $37^{\circ} \mathrm{C}$ (Fig. 2S). After removal of the unbound virus, DRG explants were overlaid with SFM and incubated at $37^{\circ} \mathrm{C}$ until the time of harvest of inoculums $(12,24$, and $36 \mathrm{~h}$ ). Inoculum of DRG explants infected with HSV-1 or mock treated was laid on Vero cells cultured in triplicate to visualize plaque formation. In order to block secondary plaque formation, human immunoglobulin G (IgG; 1:10 SigmaAldrich, St. Louis, MO) was added to the inoculums. The Vero cells were washed with PBS buffer, fixed in ethanol 


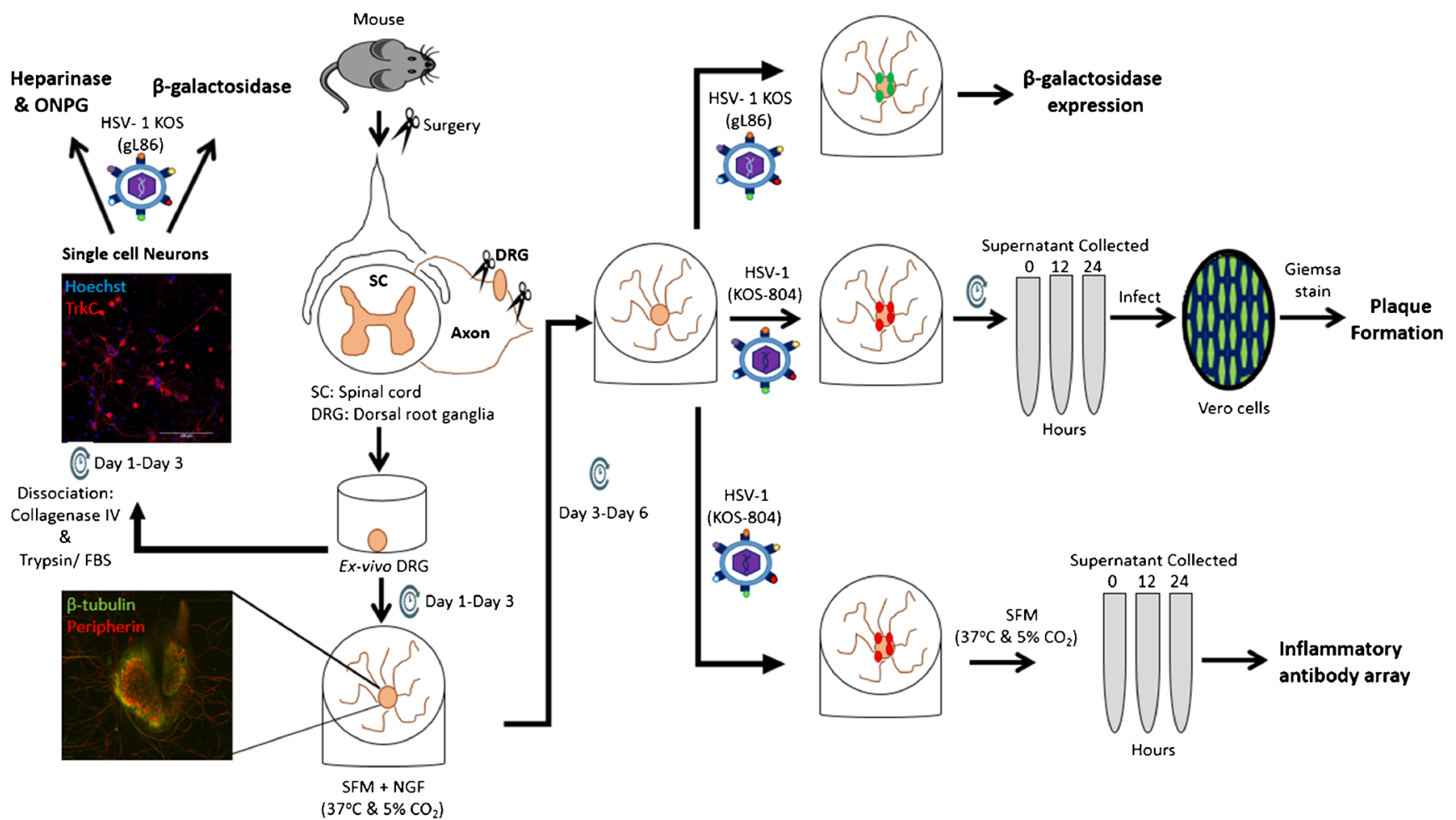

Fig. 1 Schematic representation of the experimental procedures carried out in this study. DRGs were isolated from adult NIH/Swiss mice and either plated ex vivo as a whole explant or dissociated into single cell cultures composed of neurons and satellite cells. These two models were

$80 \%$, and stained with Giemsa stain. Infectivity was quantified by counting the number of plaques.

\section{Immunofluorescence assay}

DRG explants and SCNs were either uninfected or infected with HSV-1 (KOS-804) at a concentration of 1.0 multiplicity of infection (MOI) for SCNs and 10,000 virions for explants. Samples were incubated for 1 and $12 \mathrm{~h}$ in neurobasal media under $5 \% \mathrm{CO}_{2}$ at $37^{\circ} \mathrm{C}$. After infection, they were fixed with $4 \%$ formaldehyde (Sigma-Aldrich, St. Louis, MO) for $1 \mathrm{~h}$ and washed with PBS three times for 15 min each. Explants were embedded in $3 \%$ agarose and sectioned at a thickness of $100 \mu \mathrm{m}$ using a vibratome (Pelco 100). Both sections and SCNs were incubated in primary antibodies overnight at $4{ }^{\circ} \mathrm{C}$. The following antibodies were used: anti-gD (Abcam, 1:10); anti-gB (Thermo Scientific, 1:1000); anti-TrkC (Abcam, 1:1000); anti-HS (US Biological, 1:200); anti-3OST-2 (Santa Cruz, 1:500); and Phage display derived antibody HS4C3, reactive with 3-OS HS (Ten Dam et al. 2006), $1: 10$. Primary antibodies were diluted in PBS $(0.3 \%$ Triton $X$, $0.1 \%$ sodium azide) and $1 \%$ normal serum (Vector laboratories; Burlingame, CA). The next day, DRG explants and SCNs were washed with PBS $3 \times$ for $15 \mathrm{~min}$ and incubated with secondary antibodies for $1 \mathrm{~h}$ for all conditions except the ones labeled with anti-3-OS HS HS4C3 antibody (Ten Dam et al. infected with HSV-1 KOS (gL86) reporter virus for the $\beta$-galactosidase and heparinase/ONPG assay. HSV-1 (KOS-804) replication competent virus was used for plaque formation and inflammatory antibody array

2006), which were incubated for $2 \mathrm{~h}$ in secondary antibody (anti-VSV), in the dark at room temperature. The secondary antibodies used were Alexa Fluor 488 goat anti-mouse IgG (Life Technologies, Canada) at 1:500 dilution, Cy3AffiniPure goat anti-rabbit IgG $(\mathrm{H}+\mathrm{L})$ (Li StarFish, Italy) at 1:500 dilution, donkey anti-rabbit (Invitrogen, Rockford, IL) at 1:400 dilution, donkey anti-goat (Invitrogen, Rockford, IL) at 1:400 dilution, and mouse monoclonal IgG to VSV-GVSV (P5D4, Santa Cruz, Dallas, TX) at 1:200 dilution. All other conditions that did not include anti-3-OS HS were washed with PBS $3 \times$ for $15 \mathrm{~min}$. Samples labeled with anti-3-OS HS (HS4C3) antibody were further incubated with goat antimouse Alexa Fluor 488. The samples were finally mounted on a microscope slide using Vectashield Hard Set ${ }^{\text {TM }}$ Mounting Medium with DAPI (Vector laboratories, Burlingame, CA). Samples were imaged using the Nikon Eclipse Ti A1R confocal microscope and analyzed using the NIS-Elements software.

\section{Heparanase assay}

SCNs were pre-treated with heparanase I and II (SigmaAldrich, St. Louis, MO) at 1 unit/ml for $3 \mathrm{~h}$ at $37{ }^{\circ} \mathrm{C}$ with $5 \% \mathrm{CO}_{2}$ (Tiwari et al. 2004). After pre-treatment of SCNs with heparanase and or mock treatment, the SCNs were infected with HSV-1 gL86 at 1 MOI for $2 \mathrm{~h}$ in neurobasal media 
at $37^{\circ} \mathrm{C}$ with $5 \% \mathrm{CO}_{2}$. The cells were then washed to remove any unbound viruses and were let to incubate in fresh neurobasal media overnight at $37{ }^{\circ} \mathrm{C}$ with $5 \% \mathrm{CO}_{2}$ followed by the addition of ortho-nitrophenyl- $\beta$-D-galactopyranoside (ONPG) substrate (Pierce, Rockford, IL). The $\beta$ galactosidase activity was measured at $410 \mathrm{~nm}$ wavelength using the microplate reader (PerkinElmer EnSpire: multimode plate reader).

\section{Cytokine assay}

Using a RayBio cytokine antibody array (RayBio, Norcross, GA), we assessed the expression of inflammatory markers during HSV-1 infection in mouse-derived DRG explants ex vivo (Baldwin et al. 2013). We used HSV-1 (KOS) 804 virus strain at a low concentration (10,000 virions) to infect DRG. DRGs mock infected with PBS were used as control. The supernatant of treated explants was collected after 12, 24, and $36 \mathrm{~h}$ post-infection. The cytokine antibody array was performed in accordance with the manufacturer's instructions as previously described (Baldwin et al. 2013). The expression of each cytokine was evaluated in triplicate, quantified, and normalized as per manufacturer's instructions (Fig. 4S).

\section{Results}

Susceptibility of mouse-derived DRG to HSV infection We first isolated DRGs from adult NIH/Swiss mice and established an ex vivo model to study HSV-1 infection. A group of three DRG explants plated per well in triplicate experiment was challenged in a dosage-dependent manner with reporter $\beta$-galactosidase expressing HSV-1 KOS (gL86) virions at $37^{\circ} \mathrm{C}$ or mock infected using the same culture conditions at various time points. Viral entry assay was quantified based on the amount of $\beta$-galactosidase expressed from the viral genome in which $\beta$-galactosidase expression is inducible by HSV infection (data not shown). Next, we determined HSV-1 replication by visualizing viral plaque assay (Fig. 2S). DRG explants were infected with replicationcompetent HSV-1 (KOS-804) strain (10,000 virions) or mock-infected explants at $37^{\circ} \mathrm{C}$ for $24 \mathrm{~h}$. To visualize plaque formation, Vero cells were overlaid with either the infected or the mock-infected DRG's inoculum for 12, 24, and $36 \mathrm{~h}$. The results show a larger number of plaques in a time-dependent manner. In contrast, no plaque was noticed in mock-infected DRGs (Fig. 2S).

Altogether, these results indicate the susceptibility of mouse-derived DRG ex vivo explants to HSV-1 infection.

Expression of HS and localization of $\mathrm{HSV}-1 \mathrm{gB}$ with $\mathrm{HS}$ in HSV-1-infected DRGs and SCNs We next investigated the initial stages of HSV-1 infection in DRG and SCN models.
Our focus was on HS, a widely recognized receptor involved in mediating HSV-1 attachment or binding at the host cell surface (Herold et al. 1991; Shieh et al. 1992). First, we confirmed the expression of HS in high-resolution confocal microscopy on both whole DRG tissue sections and DRGderived SCN models (Fig. 2a, b). Immunofluorescence confocal microscopy confirmed an evenly distributed expression of HS (anti-HS antibody 10E4 epitope, US Biological) among the neuronal population in the whole DRG tissue section (Fig. 2a). Similarly, a wide expression of HS was also observed in DRG-derived SCN (Fig. 2b). The isolation of primary single cell DRG neurons does not account for purification of just neuronal cells. A sub-population of satellite cells is still present. Therefore, we used neuronal specific marker TrkC to confirm the neuronal phenotype of the cell population used for this study (Fig. 2a, b).

After confirming HS expression in both models, we then infected DRGs (10,000 virions) and SCNs (1.0 MOI), with HSV-1 (KOS) 804 virus for $1 \mathrm{~h}$ at $37^{\circ} \mathrm{C}$ to visualize HSV-1 binding or attachment. As shown in Fig. 2c, d, a colocalization between HSV-1 gB and HS was clearly observed at the surface of the neuronal cell bodies in both models indicating the initial participation of HS in the event of viral attachment.

Co-localization between HSV-1 gD and 3-OS HS in both DRGs and SCNs We next evaluated if DRGs and SCNs express the modified version of HS which is 3-O-sulfated heparan sulfate (3-OS HS). The latter form of HS is a product of enzymatic modification by 3-O-sulfotransferase (3-OSTs) (Esko and Lindahl 2001). Previous studies have shown that 3OS HS, generated by 3-OST-3 isoforms, allows HSV-1 entry by interacting to HSV-1 glycoprotein D (Shukla et al. 1999). The presence of 3-OST-2 enzyme was confirmed in our neuronal models by immunofluorescence (Supplementary Fig. 3S). As indicated in Fig. 3a, b, a punctate labeling for 3-OS HS was observed on the cell surface of TrkC-positive neurons. This result provided the rationale to further examine the interaction between HSV-1 glycoprotein D $(\mathrm{gD})$ and 3-OS HS, a step essential for virus-cell fusion (Shukla et al. 1999; Tiwari et al. 2004, 2007). These experiments were conducted using whole DRGs and SCNs infected with HSV-1 or mock infected for either 1 (Fig. 3c, d) or 12 h (Fig. 3e, f), respectively. Samples were fixed and immunolabeled with antiHSV-1 gD and anti-3-OS HS (HS4C3) antibodies. As shown in Fig. 3c, d, HSV-1 gD-3-OS HS yellow co-localization at 1-h post-infection (p.i.) was observed at the surface of DRGs and SCNs, respectively. Interestingly, at later time point $(12 \mathrm{~h}$, p.i.), a similar yellow co-localization was observed in the midsection of the sliced cells in both of the models (DRGs: Fig. 3e, and SCNs: Fig. 3f). The later results suggest the role of 3-OS HS receptor in HSV-1 trafficking. 

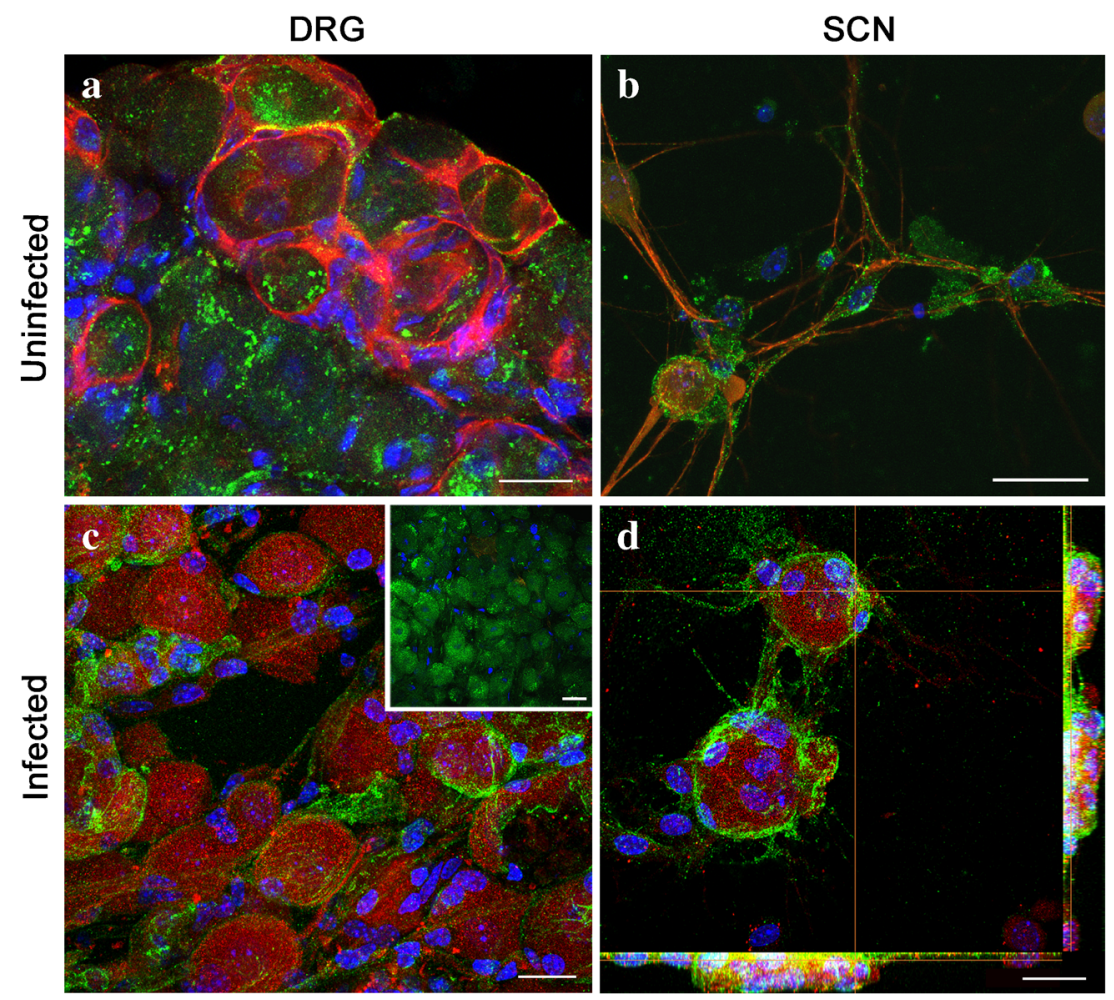

Fig. 2 Expression and localization of heparan sulfate (HS) in dorsal root ganglia (DRG) and single cell neurons (SCN). Sections of DRGs (a) and SCNs (b) were double labeled using the anti-HS 10E4 epitope FITCconjugated antibody and the specific neuronal marker against TrkC receptor showing that HS is widely expressed on the surface of DRG neurons. Samples were mounted on coverslips with mounting medium containing blue DAPI, which labels the nucleus. HSV-1-infected DRGs

\section{Enzymatic removal of cell surface HS significantly reduces} HSV-1 entry in SCNs To assess whether HS and 3-OS HS play a crucial role in virus entry, SCNs were pre-treated with enzymes heparanase I and II (1 U/ml). This enzyme selectively cleaves both heparin and HS chains containing $1 \rightarrow 4$ linkages between glucosamines and $O$-sulfated iduronic acid residues (Ernst et al. 1995). Mock-treated SCNs were used as control. Thereafter, SCNs were infected with $\beta$-galactosidase expressing HSV-1 gL86 reporter virions (1 MOI) for $2 \mathrm{~h}$ at $37^{\circ} \mathrm{C}$ and $5 \% \mathrm{CO}_{2}$. Quantification of HSV Lac $Z$ expression showed a significant $(P=0.0036)$ decrease of viral entry in the heparanase treated SCNs $(0.1691 \pm 0.006)$ compared to mocktreated $(0.2053 \pm 0.002)$ control (Fig. 4). The previous results suggest a possible role for HS and 3-OS HS in HSV-1 entry in our single neuron model.

\section{Cytokine array analysis during HSV-1 infection in DRG} explant Cytokines are a very heterogeneous family of molecules involved in inflammatory responses (Cavaillon and Haeffner-Cavaillon 1993; Newton and Dixit 2012; Mogensen 2009). Therefore, we sought to determine if DRG explants could produce inflammatory response to HSV-1 infection. A panel of more than 40 cytokines was tested in and SCNs (c, d) were then double labeled with anti-glycoprotein B (gB, red) and anti-HS marker (green). As control, a section of uninfected DRG double labeled with anti-gB and anti-HS antibodies is shown in $\mathbf{c}$, inset. Confocal imaging displayed sequestration and accumulation of HS (yellow) in the membrane region. Scale bars: $\mathbf{a}-\mathbf{d}=25 \mu \mathrm{m}$ (color figure online)

triplicate experiment in DRG explants infected with HSV-1 (KOS-804, 10,000 virions) at 12, 24, and 36 h p.i. Mockinfected DRGs were used as a control. The results of the entire panel are shown in the supplementary Fig. 4S. Interestingly, HSV-1 notably affected the expression of many members of the cytokine family at different time points. For instance, a quantitative analysis revealed a significant decrease in expression of interferon (IFN)- $\gamma$, RANTES, and TNF- $\alpha$ at $12 \mathrm{~h}$ p.i. On the contrary, other two members of the family, LIX and TIMP-2, significantly increase at $24 \mathrm{~h}$. A similar result is seen for M-CSF at $36 \mathrm{~h}$ (Fig. 5).

\section{Discussion}

In this study, we developed mouse-derived DRG explants and SCN models to investigate early stages of HSV-1 infectivity. Using confocal microscopy, we first demonstrated that diverse chains of HS (HS and 3-OS HS) are present on the surface of DRGs and SCNs as evident from the results of the immunofluorescence experiments (Figs. 2 and 3). The variability in the HS chain has been associated with the generation of binding sites for specific ligands and therefore plays a critical role 

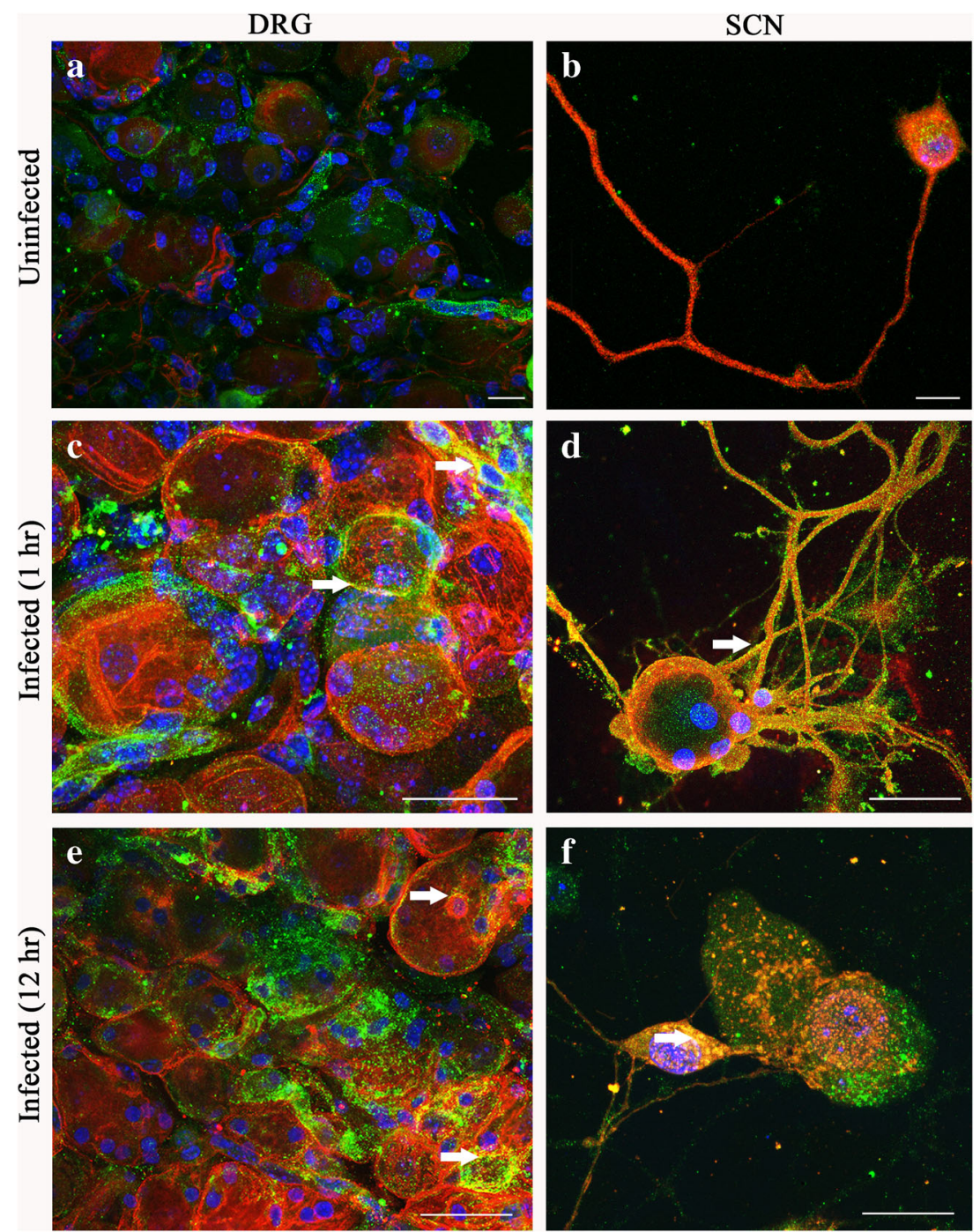

Fig. 3 Expression and localization of 3-O-sulfated heparan sulfate (3-OS $\mathrm{HS}$ ) in dorsal root ganglia (DRG) and single cell neurons (SCN). Sections of DRGs (a) and SCNs (b) were double labeled for anti-3-OS HS sulfate using the anti-HS (HS4C3) FITC-conjugated antibody and the specific neuronal marker against TrkC receptor (red) showing that 3-OS HS sulfate is widely expressed on the surfaces of the neuronal bodies. Samples were mounted on coverslips with mounting medium containing the nuclear stain blue DAPI. Both DRG tissue sections and

in maintaining and performing multiple biological functions including neuronal growth (Esko and Lindahl 2001; Mogensen and Paludan 2001; Thacker et al. 2016; Bishop et al. 2007). On the other hand, multiple pathogens including herpes viruses exploit variability in HS chain in their benefit by facilitating pathogenesis (Tiwari et al. 2012; O'Donnell and Shukla 2008). Our result established the first step of HSV-1 attachment/binding by demonstrating the colocalization between glycoprotein B (gB) to HS (Fig. 2). In addition, we also provided evidence of virus-cell fusion by colocalization between HSV-1 gD and 3-OS HS (Fig. 3). We further tested if HS and 3-OS HS were critical for HSV-1 entry in our model systems. Upon enzymatic treatment with
SCNs were then infected with HSV-1 for either $1(\mathbf{c}, \mathbf{d})$ or $12 \mathrm{~h}(\mathbf{e}, \mathbf{f})$, respectively. Double immunofluorescence with specific antibody for both HSV-1 glycoprotein gD (red) and 3-OS HS sulfate (green) revealed colocalization (yellow, arrow) of the two markers more in the neuronal cell surface at $1 \mathrm{~h}$ time point $(\mathbf{c}, \mathbf{d})$. In contrast, at $12 \mathrm{~h}$, the co-localization of the two markers (arrows) is seen more internally, in the proximity of the nuclear region (e, f). The images were taken in confocal microscopy. Scale bars: $\mathbf{a}-\mathbf{f}=25 \mu \mathrm{m}$ (color figure online)

heparanase I and II, which selectively cleaves sulfated residues in HS, a significant reduction in HSV infection was noticed in the DRG-derived SCNs suggesting the significance for HS/3-OS HS in mediating virus infection (Fig. 4).

Finally, to gain more detail insight to the post-infection inflammatory response in DRG model, a previously described chemokine array analysis was performed (Baldwin et al. 2013). We noticed significant increase (LIX, TIMP-2, and $\mathrm{M}-\mathrm{CSF}$ ) and decrease (IFN- $\gamma$, RANTES, and TNF- $\alpha$ ) in specific chemokines, which are interestingly known to influence inflammatory response either by binding directly to HS and sulfated HS or by affecting HS-dependent or HS-independent signaling pathways (Lortat-Jacob et al. 2002; Lortat-Jacob 


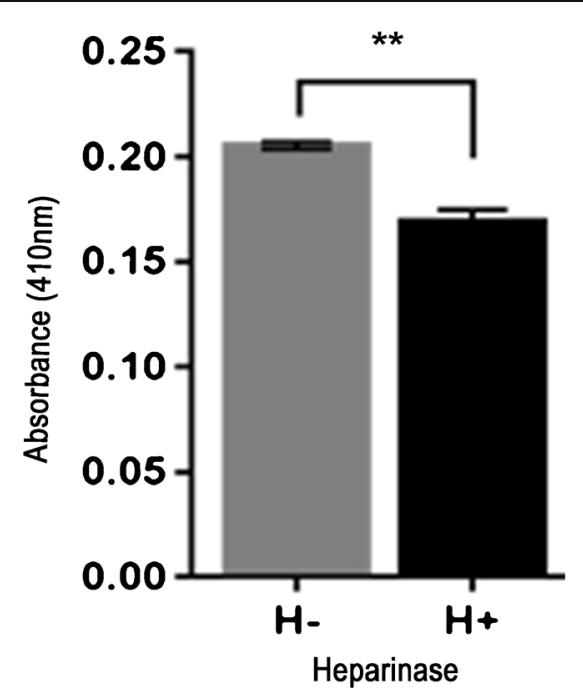

Fig. 4 Enzymatic removal of cell surface HS results significant inhibition in HSV-1 entry in DRG-derived SCNs. SCNs were treated with heparanase I and II $\left(1 \mathrm{U} / \mathrm{ml} ; \mathrm{H}^{+}\right)$or mock treated with $1 \times \mathrm{PBS}\left(\mathrm{H}^{-}\right.$ for $3 \mathrm{~h}$ at $37{ }^{\circ} \mathrm{C}$. SCNs were then challenged with $\beta$-galactosidase expressing reporter HSV-1 gL86 virus (1 MOI). After $12 \mathrm{~h}$, cells were permeabilized and incubated with ONPG substrate for quantitation of $\beta$ galactosidase activity expressed from the input viral genome. Enzymatic activity was measured $\left(\mathrm{H}^{+}\right.$sample: $0.1691 \pm 0.006 ; \mathrm{H}^{-}$sample: $0.2053 \pm 0.002$ ) by determining $\mathrm{OD}_{410}$. Data represent the mean \pm the standard deviation of results in triplicate wells in a representative experiment. The experiment was repeated three times with similar results

2009; Davis and Parish 2013; Wang and Knaut 2014). Several interesting observations were generated from our chemokine array analysis. For instance, we noticed an enhanced expression of neurospecific TIMP-2 in HSV-1-infected cell. Interestingly, an upregulation of TIMP-2 has been linked to FGF/EGF signaling that controls cell proliferation, differentiation, neurite growth (Fager and Jaworski 2000; Young et al. 2002), and markers for the ECM accumulation and fibrosis (Jaworski and Pérez-Martínez 2006; Arpino et al. 2015). The increase observed for M-CSF in our model of HSV-1 infection correlates with data previously obtained in P19 neuronal-like cells infected with HSV-1 (Smith et al. 2013; Dixit et al. 2008). In that case, as well as for other models, an enhancement of M-CSF in the brain was linked to cytoskeleton reorganization, phagocytosis, and several disease processes including neuroinflammation (Smith et al. 2013; Dixit et al. 2008). In addition, our previous finding showed a novel phagocytic uptake of HSV-1 as a primary mechanism in corneal fibroblasts derived from human eye donors (Clement et al. 2006). Further investigation will determine if M-CSF plays a major role in phagocytic uptake of HSV-1.

Similarly, an enhanced signal for LIX (CXCL5) was observed in HSV-1-infected DRG explants compared to mock infected (Fig. 5). LIX is a well-known marker for neuroinflammation, and it has been reported to be upregulated in response via a phosphatidylinositol 3-kinase (PI3 kinase) and NF-kappa B pathway. As previously reported, PI3 kinase signaling affects multiple steps during HSV entry (Tiwari and Shukla 2010).

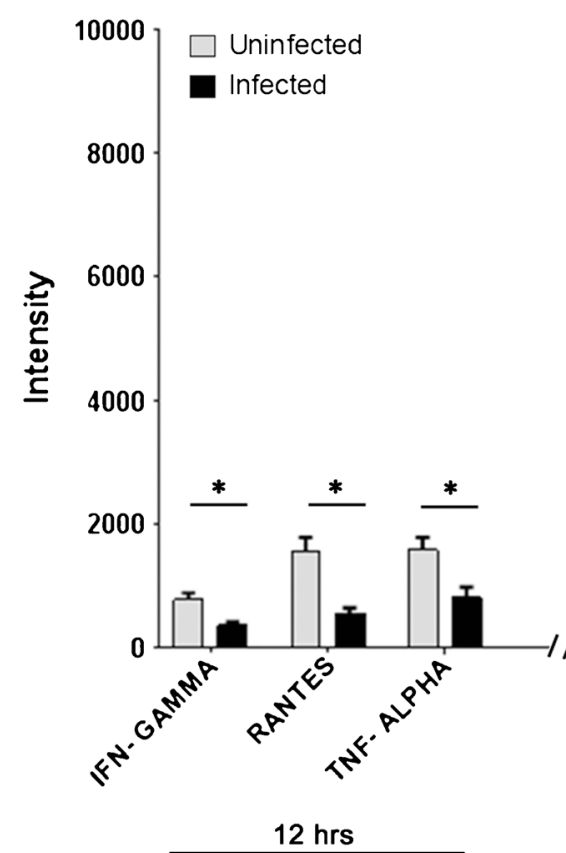

Fig. 5 Inflammatory signals in DRG explant upon HSV-1 infection. A RayBio cytokine antibody array (RayBio, Norcross, GA) as per the manufacturer's instructions was used to assess inflammatory markers produced in HSV-1 (KOS) 804 (10,000 virions) infection of ex vivo DRG explant at 12,24 , and $36 \mathrm{~h}$. The experiment was run in triplicates. Signal intensities of various inflammatory markers are plotted in

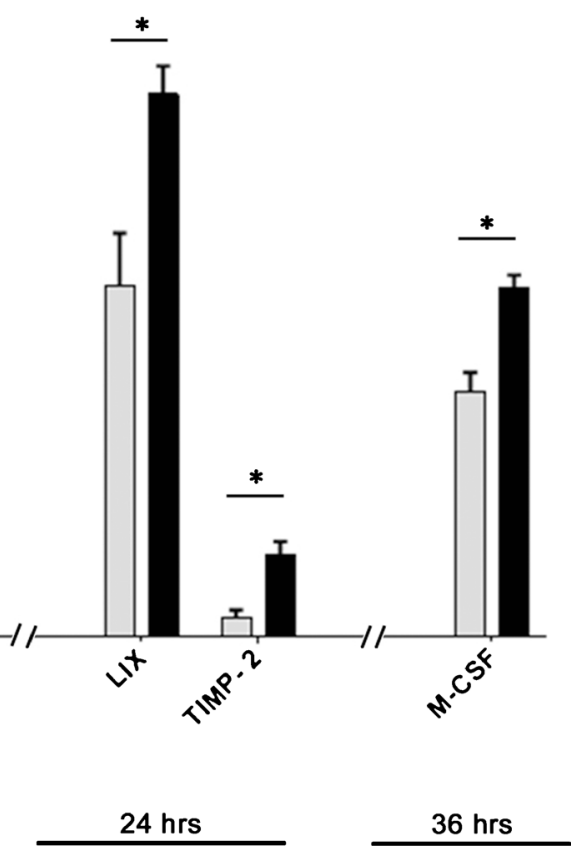

uninfected (grey bars) versus HSV-1 infected DRGs (black bars) at different time points. Inflammatory markers that resulted in significant decrease $($ IFN $-\gamma(P=0.027)$, RANTES $(P=0.011)$, and TNF- $\alpha$ $(P=0.038))$ and increase (LIX $(P=0.032)$, TIMP-2 $(P=0.013)$, and M-CSF $(P=0.011))$ in their signal intensities compared to their uninfected controls are indicated 
Finally, a significant reduction of RANTES (CCL5), TNF- $\alpha$, and IFN- $\gamma$ was observed during early phase of HSV-1 infection (Fig. 5). Interestingly, RANTES is a key player in enhancing viral binding or attachment by crosslinking virus and cell, a process that involves chemokine-HS binding (Trkola et al. 1999). The expression of TNF- $\alpha$ and IFN- $\gamma$ neuroinflammatory markers increases at $24-36 \mathrm{~h}$ (Fig. 4S) post-HSV-1 infection (Olmos and Lladó 2014).

In summary, with this study, we established ex vivo DRG explants and DRG-derived SCNs as model systems to study HSV-1 entry and associated neuroinflammation. Our data clearly suggest the involvement of HS and 3-OS HS in mediating HSV-1 infection. Further investigations are required to address which inflammatory cytokine are specifically induced under which sub-set of sulfated HS (Lawrence et al. 2007). These findings, together with additional information on the critical epitopes involved during HSV-1 gD interaction with 3-OS HS, will advance our current understanding on the HSV1-induced pathogenesis, neuroinflammation, and tissue damage. The results of these experiments will be extremely useful for the development of the novel therapeutic interventions.

Acknowledgements This research investigation was supported by the Midwestern University (MWU) Intramural grant funding to V.T. and M.F. C.S. was supported by the Biomedical Master program from College of Health Sciences.

Compliance with ethical standards In accordance with institutional review board-approved protocols (IACUC-Midwestern University), mouse-derived DRG explants were isolated from adult NIH/Swiss mice (Harlan Laboratories, Chicago, IL).

Conflict of interest The authors declare that they have no conflict of interest.

Open Access This article is distributed under the terms of the Creative Commons Attribution 4.0 International License (http:// creativecommons.org/licenses/by/4.0/), which permits unrestricted use, distribution, and reproduction in any medium, provided you give appropriate credit to the original author(s) and the source, provide a link to the Creative Commons license, and indicate if changes were made.

\section{References}

Antoine TE, Park PJ, Shukla D (2013) Glycoprotein targeted therapeutics: a new era of anti-herpes simplex virus-1 therapeutics. Rev Med Virol 23(3):194-208. doi:10.1002/rmv.1740

Arpino V, Brock M, Gill SE (2015) The role of TIMPs in regulation of extracellular matrix proteolysis. Matrix Biol 44-46: $247-254$

Baldwin J, Park PJ, Zanotti B, Maus E, Volin MV, Shukla D, Tiwari V (2013) Susceptibility of human iris stromal cells to herpes simplex virus 1 entry. J Virol 87(7):4091-4096. doi:10.1128 /JVI.03235-12

Bishop JR, Schuksz M, Esko JD (2007) Heparan sulphate proteoglycans fine-tune mammalian physiology. Nature 446(7139):1030-1037
Cavaillon JM, Haeffner-Cavaillon N (1993) Cytokines and inflammation. Rev Prat 43(5):547-552

Clement C1, Tiwari V, Scanlan PM, Valyi-Nagy T, Yue BY, Shukla DJ (2006) A novel role for phagocytosis-like uptake in herpes simplex virus entry. Cell Biol 174(7):1009-1021

Connolly SA, Jackson JO, Jardetzky TS, Longnecker R (2011) Fusing structure and function: a structural view of the herpesvirus entry machinery. Nat Rev Microbiol 9(5):369-381. doi:10.1038 /nrmicro2548

Davis DAS, Parish CR (2013) Heparan sulfate: a ubiquitous glycosaminoglycan with multiple roles in immunity. Front Immunol 4:470. doi:10.3389/fimmu.2013.00470 Published online 2013 Dec 18

Dixit R, Tiwari V, Shukla D (2008) Herpes simplex virus type 1 induces filopodia in differentiated P19 neural cells to facilitate viral spread. Neurosci Lett 440(2):113-118 . doi:10.1016/j.neulet.2008.05.03 Published online 2008 May 15

Eisenberg RJ, Atanasiu D, Cairns TM, Gallagher JR, Krummenacher C, Cohen GH (2012) Herpes virus fusion and entry: a story with many characters. Viruses 4(5):800-832

Ernst S, Langer R, Cooney CL, Sasisekharan R (1995) Enzymatic degradation of glycosaminoglycans. Crit Rev Biochem Mol Biol 30: $387-444$

Esko JD, Lindahl U (2001) Molecular diversity of heparan sulfate. J Clin Invest 108(2):169-173 Review. No abstract available

Fager N, Jaworski DM (2000) Differential spatial distribution and temporal regulation of tissue inhibitor of metalloproteinase mRNA expression during rat central nervous system development. Mech Dev 98:105-109

Ferro V, Dredge K, Liu L, Hammond E, Bytheway I, Li C, Johnstone K, Karoli T, Davis K, Copeman E, Gautam A (2007) PI-88 and novel heparan sulfate mimetics inhibit angiogenesis. Semin Thromb Hemost 33(5):557-568

Fraser NW, Valyi-Nagy T (1993) Viral, neuronal and immune factors which may influence herpes simplex virus (HSV) latency and reactivation. Microb Pathog 15(2):83-91

Fueshko S, Wray S (1994) LHRH cells migrate on peripherin fibers in embryonic olfactory explant cultures: an in vitro model for neurophilic neuronal migration. Dev Biol 166(1):331-348

Geraghty RJ, Krummenacher C, Cohen GH, Eisenberg RJ, Spear PG (1998) Entry of alphaherpesviruses mediated by poliovirus receptor-related protein 1 and poliovirus receptor. Science 280(5369): $1618-1620$

Hadigal S, Shukla D (2013) Exploiting herpes simplex virus entry for novel therapeutics. Viruses. 5(6):1447-1465. doi:10.3390 /v5061447

Hadigal SR, Agelidis AM, Karasneh GA, Antoine TE, Yakoub AM, Ramani VC, Djalilian AR, Sanderson RD, Shukla D (2015) Heparanase is a host enzyme required for herpes simplex virus-1 release from cells. Nat Commun 6:6985

Herold BC, WuDunn D, Soltys N, Spear PG (1991) Glycoprotein C of herpes simplex virus type 1 plays a principal role in the adsorption of virus to cells and in infectivity. J Virol 65:1090-1098

Jaworski DM, Pérez-Martínez L (2006) Tissue inhibitor of metalloproteinase-2 (TIMP-2) expression is regulated by multiple neural differentiation signals. J Neurochem 98(1):234-247. doi:10.1111/j.1471-4159.2006.03855.x

Joyce JA, Freeman C, Meyer-Morse N, Parish CR, Hanahan D (2005) A functional heparan sulfate mimetic implicates both heparanase and heparan sulfate in tumor angiogenesis and invasion in a mouse model of multistage cancer. Oncogene 24(25):4037-4051

Lawrence R, Yabe T, Hajmohammadi S, Rhodes J, McNeely M, Liu J, Lamperti ED, Toselli PA, Lech M, Spear PG, Rosenberg RD, Shworak NW (2007) The principal neuronal gD-type 3-O- 
sulfotransferases and their products in central and peripheral nervous system tissues. Matrix Biol 26(6):442-455

Li JP, Vlodavsky I (2009) Heparin, heparan sulfate and heparanase in inflammatory reactions. Thromb Haemost 102(5):823-828. doi:10.1160/TH09-02-0091

Little SP, Schaffer PAA (1981) Expression of the syncytial (syn) phenotype in HSV-1, strain KOS: genetic and phenotypic studies of mutants in two syn loci. Virology 112:686-702

Lortat-Jacob H (2009) The molecular basis and functional implications of chemokine interactions with heparan sulphate. Curr Opin Struct Biol 19(5):543-548. doi:10.1016/j.sbi.2009.09.003

Lortat-Jacob H, Grosdidier A, Imberty A (2002) Structural diversity of heparan sulfate binding domains in chemokines. Proc Natl Acad Sci U S A 99(3):1229-1234

Mogensen TH (2009) Pathogen recognition and inflammatory signaling in innate immune defenses. Clin Microbiol Rev 22(2):240-273. doi:10.1128/CMR.00046-08

Mogensen TH, Paludan SR (2001) Molecular pathways in virus-induced cytokine production. Microbiol Mol Biol Rev 65(1):131-150. doi:10.1128/MMBR.65.1.131-150.2001

Montgomery RI, Warner MS, Lum BJ, Spear PG (1996) Herpes simplex virus-1 entry into cells mediated by a novel member of the TNF/ NGF receptor family. Cell 87:427-436

Newton K, Dixit VM (2012) Signaling in innate immunity and inflammation. Cold Spring Harb Perspect Biol 4(3):a006049. doi:10.1101 /cshperspect.a006049

Nicoll MP, Proença JT, Efstathiou S (2012) The molecular basis of herpes simplex virus latency. FEMS Microbiol Rev 36(3):684-705

O'Donnell CD, Shukla D (2008) The importance of heparan sulfate in herpesvirus infection. Virol Sin 23(6):383-393

Olmos G, Lladó J (2014) Tumor necrosis factor alpha: a link between neuroinflammation and excitotoxicity. Mediat Inflamm 2014: 861231. doi: $10.1155 / 2014 / 861231$

Parish CR (2006) The role of heparan sulphate in inflammation. Nat Rev Immunol 6(9):633-643

Raman K, Karuturi R, Swarup VP, Desai UR, Kuberan B (2012) Discovery of novel sulfonated small molecules that inhibit vascular tube formation. Bioorg Med Chem Lett 22(13):4467-4470. doi:10.1016/j.bmcl.2012.04.014

Roizman B, Whitley RJ (2013) An inquiry into the molecular basis of HSV latency and reactivation. Annu Rev Microbiol 67:355-374. doi:10.1146/annurev-micro-092412-155654

Rusnati M, Oreste P, Zoppetti G, Presta M (2005) Biotechnological engineering of heparin/heparan sulphate: a novel area of multi-target drug discovery. Curr Pharm Des 11(19):2489-2499

Shieh MT, WuDunn D, Montgomery RI, Esko JD, Spear PG (1992) Cell surface receptors for herpes simplex virus are heparan sulfate proteoglycans. J Cell Biol 116(5):1273-1281

Shukla D, Spear PG (2001) Herpesviruses and heparan sulfate: an intimate relationship in aid of viral entry. J Clin Invest 108:503-510 doi: 13799

Shukla D, Liu J, Blaiklock P, Shworak NW, Bai X, Esko JD, Cohen GH, Eisenberg RJ, Rosenberg RD, Spear PG. 1999. A novel role for 3-Osulfated heparan sulfate in herpes simplex virus 1 entry. Cell 99:1322

Simmons A (2002) Clinical manifestations and treatment considerations of herpes simplex virus infection. J Infect Dis 186(Suppl 1):S71S77

Smith AM, Gibbons HM, Oldfield RL, Bergin PM, Mee EW, Curtis MA, Faull RLM, Dragunow M (2013) M-CSF increases proliferation and phagocytosis while modulating receptor and transcription factor expression in adult human microglia. J Neuroinflammation 10:85 . doi:10.1186/1742-2094-10-85 Published online 2013 Jul 17

Spear PG (2004) Herpes simplex virus: receptors and ligands for cell entry. Cell Microbiol 6(5):401-410

Ten Dam GB, Kurup S, van de Westerlo EM, Versteeg EM, Lindahl U, Spillmann D, van Kuppevelt TH (2006) 3-O-sulfated oligosaccharide structures are recognized by anti-heparan sulfate antibody HS4C3. J Biol Chem 281(8):4654-4662

Thacker BE, Seamen E, Lawrence R, Parker MW, Xu Y, Liu J, Vander Kooi CW, Esko JD (2016) Expanding the 3-O-sulfate proteomeenhanced binding of neuropilin-1 to 3-O-sulfated heparan sulfate modulates its activity. ACS Chem Biol 11(4):971-980. doi:10.1021/acschembio.5b00897

Tiwari V, Shukla D (2010) Phosphoinositide 3 kinase signalling may affect multiple steps during herpes simplex virus type- 1 entry. J Gen Virol 91(Pt 12):3002-3009. doi:10.1099/vir.0.024166-0

Tiwari V, Clement C, Duncan MB, Chen J, Liu J, Shukla D (2004) A role for 3-O-sulfated heparan sulfate in cell fusion induced by herpes simplex virus type 1. J Gen Virol. 85(Pt 4):805-809

Tiwari V, ten Dam GB, Yue BY, van Kuppevelt TH, Shukla D (2007) Role of 3-O-sulfated heparan sulfate in virus-induced polykaryocyte formation. FEBS Lett 581(23):4468-4472

Tiwari V, Liu J, Valyi-Nagy T, Shukla D (2011) Anti-heparan sulfate peptides that block herpes simplex virus infection in vivo. J Biol Chem 286(28):25406-25415. doi:10.1074/jbc.M110.201103

Tiwari V, Maus E, Sigar IM, Ramsey KH, Shukla D (2012) Role of heparan sulfate in sexually transmitted infections. Glycobiology 22(11):1402-1412. doi:10.1093/glycob/cws106

Tiwari V, Tarbutton MS, Shukla D (2015) Diversity of heparan sulfate and HSV entry: basic understanding and treatment strategies. Molecules 20(2):2707-2727. doi:10.3390/molecules20022707

Trkola A, Gordon C, Matthews J, Maxwell E, Ketas T, Czaplewski L, Proudfoot AEI, Moore JP (1999) The CC-chemokine RANTES increases the attachment of human immunodeficiency virus type 1 to target cells via glycosaminoglycans and also activates a signal transduction pathway that enhances viral infectivity. J Virol 73(8): 6370-6379

Wang J, Knaut H (2014) Chemokine signaling in development and disease. Development 141(22):4199-4205. doi:10.1242/dev.101071

Whitley RJ, Roizman B (2001) Herpes simplex virus infections. Lancet 357(9267):1513-1518

Wilson AC, Mohr I (2012) A cultured affair: HSV latency and reactivation in neurons. Trends Microbiol 20(12):604-611. doi:10.1016/j. tim.2012.08.005

WuDunn D, Spear PG (1989) Initial interaction of herpes simplex virus with cells is binding to heparan sulfate. J Virol 63(1):52-58

Yabe T, Maeda N (2015) Histochemical analysis of heparan sulfate 3-Osulfotransferase expression in mouse brain. Methods Mol Biol 1229: 377-387. doi:10.1007/978-1-4939-1714-3 29

Yabe T, Hata T, He J, Maeda N (2005) Developmental and regional expression of heparan sulfate sulfotransferase genes in the mouse brain. Glycobiology 15(10):982-993

Young DA, Phillips BW, Lundy C, Nuttall RK, Hogan A, Schultz GA, Leco KJ, Clark IM, Edwards DR (2002) Identification of an initiator-like element essential for the expression of the tissue inhibitor of metalloproteinases-4 (Timp-4) gene. Biochem J 364:89-99

Zhang X, Wang B, Li JP (2014) Implications of heparan sulfate and heparanase in neuroinflammation. Matrix Biol 35:174-181. doi:10.1016/j.matbio.2013.12.009 